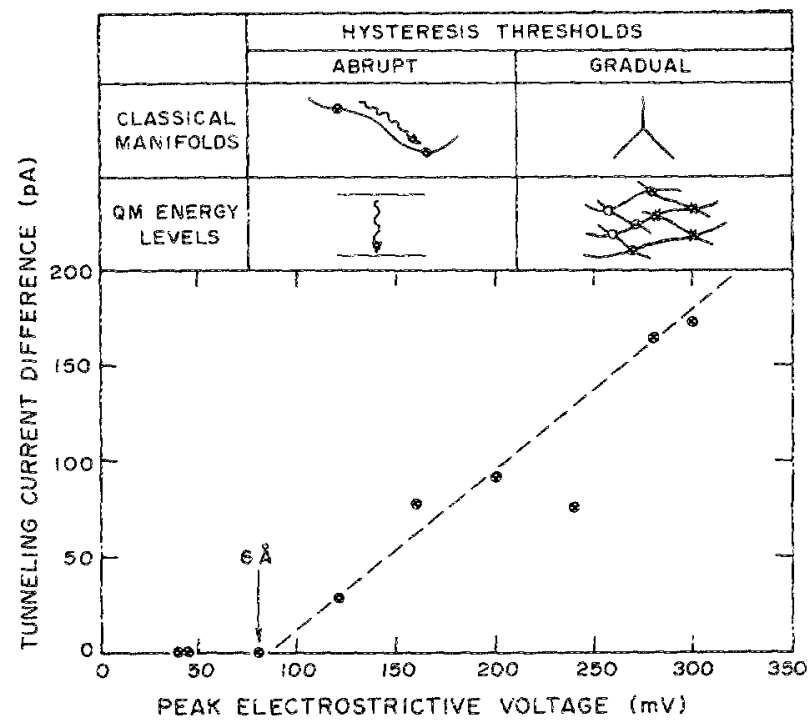

FIG. 4. Threshold variation of hysteresis. The ordinate indicates the difference in tunneling current between the beginning and end of the stress-strain cycles. The corresponding maximum voltage variations are plotted along the abscissa. The insets illustrate various types of hysteresis thresholds.

between two eigenstates of the system. Barkhausen jumps in ferromagnets ${ }^{1}$ and domain realignments in ferroelectrics ${ }^{5}$ are examples of this type of hysteresis threshold.

Alternatively, the gradual onset of irreversible behavior can be modeled classically by sequences of transitions across quasi-reversible forks or bifurcations in the phase space trajectories. "This situation is represented by the "Y" pattern in Fig. 4. The quantum-mechanical counterpart of these junction sequences are level crossings which in complex multiparameter systems can form networks that connect the ei- genstates." ${ }^{9.10}$ Since the Dyson-Lenard results on the stability of matter are essentially existence theorems concerned only with the quantum mechanical ground state, ${ }^{11}$ it is evident that hysteresis threshold measurements can yield further information regarding the coupling of the ground state to higher levels by microscopic stress fields. ${ }^{12,13}$

In summary, this experiment demonstrates the feasibility of using STM technology to investigate the transition from reversible to irreversible behavior in mechanical systerns. It would be interesting to replace the current piezoelectric devices with single crystals that exhibit strain when subjected to electro- or magnetostrictive stresses. It would also be interesting to apply this technique to materials of technical importance such as steel and keviar composites.

We are grateful to Professor A. M. Goldman for assistance with all aspects of this work. It is also a pleasure to acknowledge the support of the Research Corporation.

'H. Weinstock, T. Erber, and M. Nisenoff, Phys. Rev. B 31, 1535 (1985).

${ }^{2}$ T. Erber and S. A. Guralnick, Ann. Phys. (N.Y.) 181, 25 (1988).

${ }^{3}$ G. Binnig and D. P. E. Smith, Rev. Sci. Instrum. 57, 1688 (1986).

${ }^{4}$ A. P. Fein, J. R. Kirtiey, and R. M. Feenstra, Rev. Sci. Instrum. 58, 1806 (1987).

'D. A. Berlincourt, D. R. Curran, and H. Jaffe, in Physical Acoustics, edited by W. P. Mason (Academic, New York, 1964), Vol. 1, Part A, pp. 169 270

6H. Kaizuka and B. Siu. Jpr. J. Appl. Phys. 27, L-773 (1988).

'H. J. Mamin, E. Ganz, D. W. Abraham, R. E. Thomson, and J. Clarke, Phys. Rev. B 34, 9015 (1986).

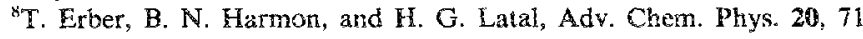
(1971).

9. von Neumann and E. Wigner, Phys. Zeit. 30, 467 (1929).

"W. 3. Caspers, Physica \$0, 125 (1968).

${ }^{12 E . ~ H . ~ L i e b, ~ R e v . ~ M o d . ~ P h y s, ~ 48, ~} 553$ (1976).

'O. H. Nielsen and R. M. Martin, Phys. Rev. B 32, 3780 (1985).

1.3. J. J. Godfrey, Phys. Rev. B 37, 10176 (1988).

\title{
Two-band modeling of narrow band gap and interband tunneling devices
}

\author{
J.R. Söderström, a) E.T. Yu, M.K. Jackson, Y. Rajakarunanayake, and T. C. McGill \\ T. J. Watson, Sr., Laboratory of Applied Physics, California Institute of Technology, \\ Pasadena, California 91125
}

(Received 30 November 1989; accepted for publication 2 Aprii 1990)

\begin{abstract}
A two-band transfer matrix method has been developed to study tumeling currents in narrow gap and interband tunnel structures. This relatively simple model gives good agreement with recently reported experimental results for InAs/AISb/hnAs/AlSb/InAs double-barrier heterostructures and InAs/AlSb/GaSb/AlSb/InAs resonant interband tunneling devices, and should be useful in the design of new interband tunneling devices.
\end{abstract}

The double-barrier resonant tunneling diode proposed by Tsu and Esaki has been a subject of great interest over the past several years due to its potential for use in applications such as high-frequency oscillators ${ }^{2}$ and digital logic. ${ }^{3}$ Until

\footnotetext{
"Present address: Chalmers University of Technology, Department of Physics, S-41296 Göteborg, Sweden.
}

recently, most of the work has been in the GaAs/AlGaAs material system, mainiy because of availability of high-quality materials grown by molecular beam epitaxy (MBE) or metalorganic chemical vapor deposition (MOCVD). However, recent progress in the growth of other materials with different band gaps and band offets has stimulated the development of novei tunnel structures. With the freedom to 
choose diferent band lineups, devices based on $\ln \mathrm{As} / \mathrm{AlSb}$ or InAs/ZnTe in which electrons tunnel through barriers at energies near the valence-band edge have been proposed and demonstrated. ${ }^{4-6}$ Recently, resonant interband tunneling (RT) in InAs/AiSb/GaSb/AISb/InAs heterostructures, in which tunneling takes place through resonant valenceband levels, has indepencentiy been proposed ${ }^{7}$ and demonstrated, ${ }^{8}$ showing very high peak-to-valley current ratios. Analysis of these new structures, based on narrow band-gap materials and neariy broken or completely broken-gap (sype 11) band alignments, requires the use of models that incorporate both the valence and the conduction bands.

In this communication we present calculations of current-voltage characteristics in narrow gap and broken gap tunnel structures, using a two-band model which explicitly includes the conduction and light-hole bands. A two-band model has been applied previously to caiculate the transmission coefficient in an InGaAs/GaSbAs tunnel structure." We have used a two-band model due to Bastard ${ }^{10,11}$ to develop a transfer matrix approach ${ }^{2}$ to tumeling in heterostructures. This is combined with realistic band-bending calculations to predict semiquantitatively behavior of peak voltages and currents observed experimentally in these structures. The ability to model this new class of tunneling devices has also allowed us to calculate current-voltage characteristics for an InAs/GaSb/InAs resonan interband tunnel structure. In this device, negative differential resistance is based on resonant tunnelng through a confined valence-band state in the Gasb layer.

The two band model used has been described previousiy by Bastard." The heavy-hole band is neglected in the calculations, based on the weak coupling between conduction and heavy-hole bands. The model is based on an effective Hamitonian in which the wave function is composed of both conduction-and valence-band components. This leads to an effective Schrödinger equation for the two components,

$$
\left(\begin{array}{cc}
E_{\mathrm{c}}-E & \sqrt{\frac{2}{3}} \Pi n k \\
\sqrt{\frac{2}{3}} \Pi \hbar k & E_{u}-E
\end{array}\right)\left(\begin{array}{l}
\Psi_{c} \\
\Psi_{v}
\end{array}\right)=0,
$$

from which the dispersion relation $E(k)$ can be ootained. The real parameter $I$ refects the strength of the coupling between the valence and conduction bands; a single value of $I$ is used for all materials. $E_{c}, E_{v}$, and $k$ are the conductionband-edge energy, valence-band-edge energy, and wave vector, respectively. $\Psi_{c}$ and $\Psi_{u}$ are the conduction- and valence-band components of the total wavefunction, respectively.

Boundary conditions require continuity of the conduction-and valence-band components of the wavefunction, and of the probability currents associated with each. These conditions allow us to relate the components of the wavefunction across an interface, or across a region with constant band edge potential. Following the general approach of the one-band transfer matrix method developed by $\mathrm{Kane}{ }^{12}$ we choose the wavefunction to be composed of plane wave components traveling in the positive and regative $z$ directions, where $z$ is the growth direction. In layer n (numbering layers starting with the injecting layer) the wave function is then given by

$$
\left(\begin{array}{c}
\Psi_{c} \\
\Psi_{i}
\end{array}\right)=\left(\begin{array}{l}
A_{c, n} e^{i k_{n^{2}}}+B_{c, n} e^{-i k_{n^{z}}} \\
A_{u, n} e^{i k_{n^{z}}}+B_{u, n} e^{-i k_{n^{z}}}
\end{array}\right)
$$

The transfer matrix for an arbitrary heterostructure is obtained by nultiplying the individual transfer matrices for all of the layers composing the heterostructure. This approach facilitates calculations in the presence of an electric field along the growth direction, since the heterostructure can be approximated by a series of thin layers, each at constant potential, whose band edge positions follow the profiles obtained from band-bending calculations. The final transmission coefficient for the heterostructure is obtained from the simple ratio of the incoming and transmitted probability currents:

$$
\begin{aligned}
T= & \frac{k_{N}}{k_{i}}\left[\left(\frac{\left|A_{c, N}\right|^{2}}{E-E_{v, N}}+\frac{\left|A_{v, N}\right|^{2}}{E-E_{c, N}}\right) /\right. \\
& \left.\left(\frac{\left|A_{c, 1}\right|^{2}}{E-E_{i, 1}}+\frac{\left|A_{v, 1}\right|^{2}}{E-E_{c, 1}}\right)\right]
\end{aligned}
$$

where layers 1 and $N$ are the injecting and collecting contact layers, respectively. The relation between $A_{c, N}$ and $A_{v, N}$ in the final layer, where the wave function is oniy outgoing, is obtained from Eq. (1).

Band bending in the heterostructure is calculated using the Thomas-Femi approximation, assuming a zero-temperature electron distribution. The current at a given voltage is calculated by integrating the transmission coefficient over the incoming electron distribution, incorporating the appropriate velocities and Fermi factors at finite temperature in the injecting and collecting contacts. In the calculation of the current, it is necessary to in tegrate over wave vectors parallel and perpendicular to the interface. In the integration over the parallel wave vectors, the transmission coefficient is calculated using the two-band model described above, for a band profle that varies with parallel wave vector as given by the bulk dispersion relations.

In order to illustrate this simple model, we present in Fig. 1 (a) the band profie for an InAs/AISb double-barrier heterostructure. The barrier and well thicknesses are 27 and $66 \AA$, respectively, corresponding to the structure reported in Ref. 5. The doping proflile used was the same as in Ref. 5 . $A$ vaience-band offet of $60 \mathrm{meV}$ between in $\mathrm{As}$ and $\mathrm{A} 1 \mathrm{Sb}$ was used. Although no direct measurements of the $\operatorname{InAs} / \mathrm{AlSb}$ and ofiset have been made, this value is consistent, within experimental uncertainties, with previous reports of the GaSb/AlSb (Ref. 13) and InAs/GaSb (Refs. 14 and 15) valence-band offets, and the assumption of transitivity. The calculation was performed for a temperature of 77 . The agreement of the calculated peak current and peak voltage with the experimental results is good. There are two features in the calculated current-voltage curve of Fig. 1 (b). The large peak at lower bias corresponds to electron tunneling from the conduction-band continumm in the emitter; the smaller shoulder at higher bias is due to tunneling involving the quantum state confined in the emitter accumulation layer. In the experimental current-voltage curve of Fig. $1(\mathrm{c}) \mathrm{a}$ similar plateau is seen at voltages past resonance. However, circuit instabilities in this region affect the observed charac- 

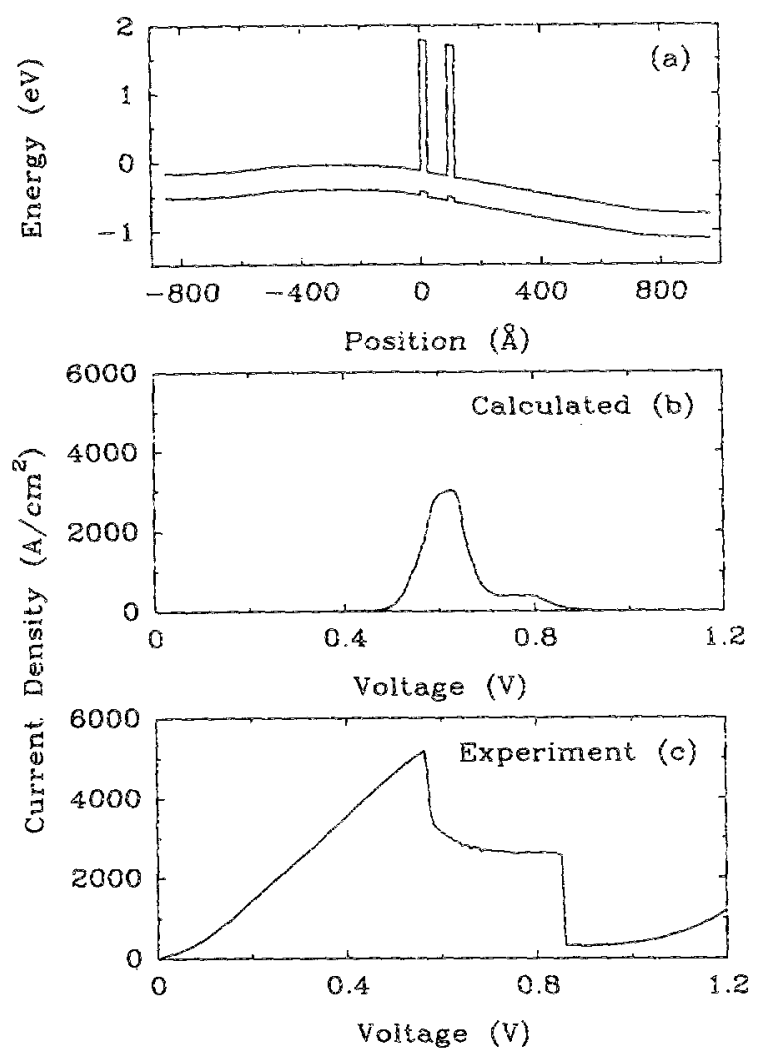

FIG. 1. Results for an InAs/AISb double-barrier structure, (a) Band profile calculated for a bias of $0.6 \mathrm{~V}$. The zero of energy is the Fermi level in the left electrode. (b) Calculated current-voltage behavior for $T=77 \mathrm{~K}$. (c) Experimentally observed current voltage characteristic at $T^{\prime}=77 \mathrm{~K}$ (from Ref. 5).

teristics; detailed comparisons of the shapes of the caiculated and observed current-voltage characteristics in the negative differential resistance region are probably not meaningful.

The agreement between theory and experiment obtained with our two-band model calculation would not have been obtained with a one-band calculation, even though the funneling is only through confined conduction-band states, because the electrons tunnel through the AlSb barriers with energies very close to the valence-band edge. It is necessary to incorporate the coupling of the conduction-and valenceband states in any model used to study these structures. Although our simple calculation neglects the effects of the heavy holes, it gives good agreement with the experimental results, and shows its utility for these types of heterostructures.

The two-band calculation is also necessary in studying broken-gap structures, involving interband tunneling. Electron resonances with valence-band states are essential in such structures, and cannot be treated with the simple oneband model. In Fig. 2(a) we show the band profile of the InAs/AISb/GaSb/AISb/InAs RIT structure reported in Ref. 8. The doping profile and the $24-\AA$ AlSb and $63-\AA \mathrm{GaSb}$ layer thicknesses used in the calculation are the same as in Ref. 8. A value of $400 \mathrm{meV}$ is used for the $\mathrm{GaSb} / \mathrm{AlSb}$ valence-band offset. ${ }^{13}$ The calculated current-voltage characteristics are shown in Fig. 2(b). The valence-band offset between InAs/GaSb of $460 \mathrm{meV}$ was chosen in order to
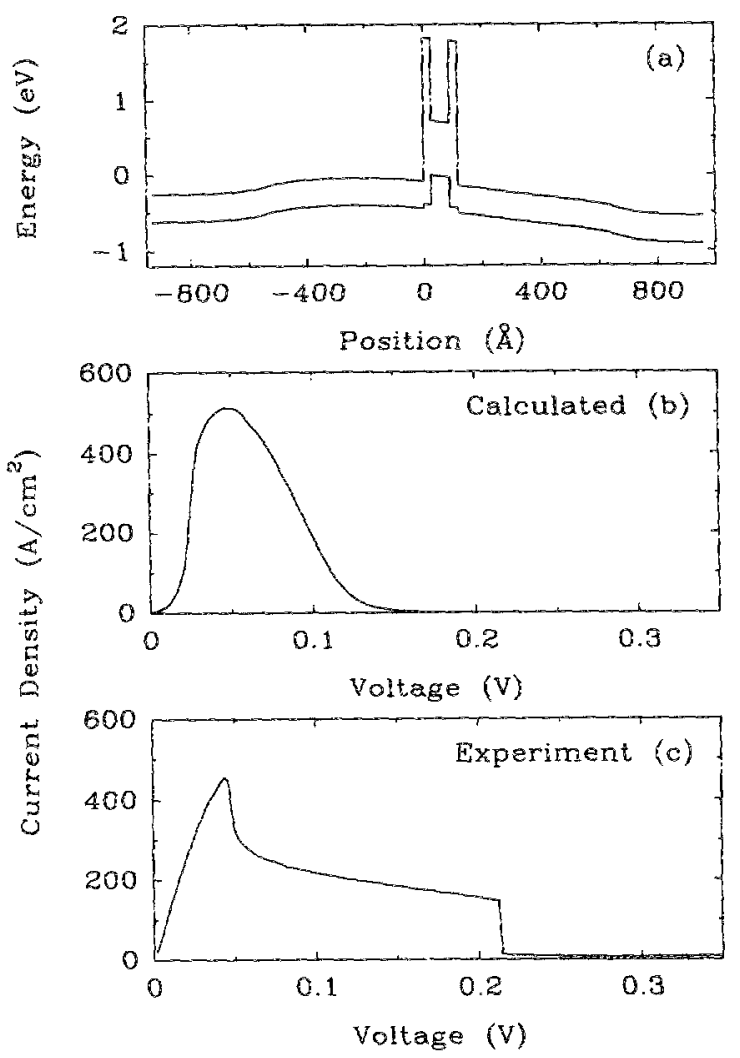

FIC. 2. Results for an InAs/AISb/GaSb/A1Sb/InAs resonant interband tunneling (RIT) structure. (a) Band profile calculated for a bias of $0.3 \mathrm{~V}$. The zero of energy is the Fermi level in the left electrode. (b) Calculated current-voltage behavior for $T=77 \mathrm{~K}$. (c) Experimentally observed current voltage characteristic at $T=77 \mathrm{~K}$ (from Ref. 8 )

match the experimental peak voltage seen in Fig. 2(c). This value is within the experimental uncertainty of previous reports ${ }^{14,15}$ of $510-560 \mathrm{meV}$. As can be seen from Fig, 2, the agreement of the peak current predicted by the theory with the experimental value is very good, with the In As/GaSb valence-band offset being the only adjustable parameter.
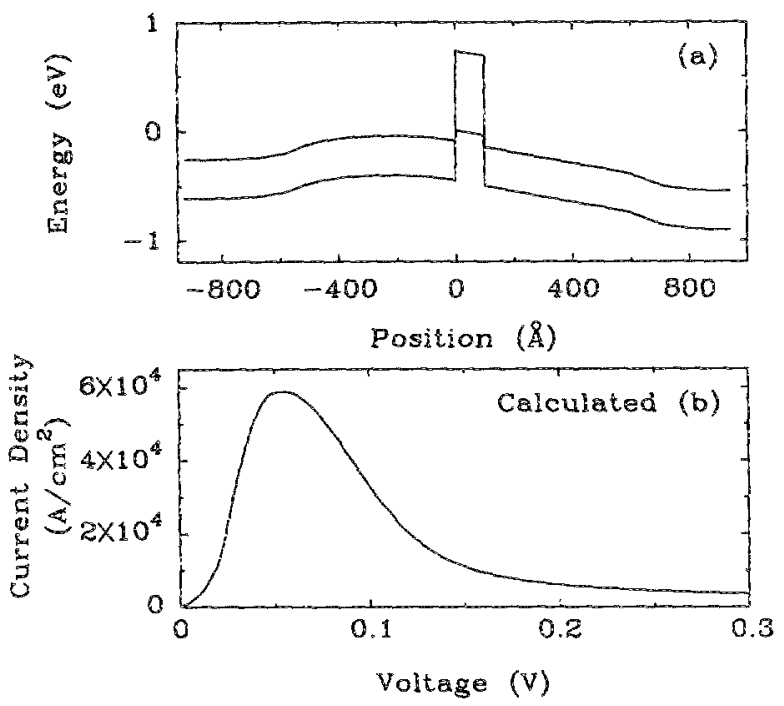

FIG. 3. Results for an InAs/GaSb/InAs resonant interband tumneling (RIT) structure. (a) Band profile calculated for a bias of $0.3 \mathrm{~V}$. The zero of energy is the Fermi hevel in the left electrode. (b) Calculated current-voltage behavior for $T=77 \mathrm{~K}$. 
Having established that this model can predict with resonable accuracy the characteristics of the InAs/AlSb double barrier and of the InAs/AlSb/GaSb/AlSb/InAs RT structure, we now study a novel InAs/GaSb/InAs RIT structure, which has recently been denonstrated. ${ }^{18}$ In Fig. 3 (a) we show the band profile of the device, calculated with a GaSb layer thickness of $100 \AA$; the doping profile is the same as for the RIT structure reported in Ref. 8. In Fig. 3(b) we show the calculated current-voltage characteristics. At the chosen GaSb layer thickness the alignment of the valence-band resonant level in the GaSb with the injecting electron energy is such that a peak current on the order of $6 \times 10^{4}$ $\mathrm{A} / \mathrm{cm}^{2}$ at a voltage of $0.05 \mathrm{~V}$ is predicted. The quasibound state If ifetime estimated from the transmission resonance full width at half-maximum (FWHM) by $\tau=\tilde{n} / \Delta E_{\mathrm{FWHM}}$ is 50 fs, suggesting that this device could be useful in high-frequency oscillator and switching circuits.

In summary, we have developed a two-band transfer matrix method for studying the tunneling currents in narrow gap and interband tunnel structures. The relatively simple model gives good agreement with reported experimental results for an InAs/AISb double barrier, and an InAs/A1So/ $\mathrm{GaSb} / \mathrm{AlSb} / \mathrm{InAs}$ resonant interband tunneling (RIT) structure. We have also studied an InAs/GaSb/InAs RIT device, in which we calculate a peak current of $6 \times 10^{4} \mathrm{~A} /$ $\mathrm{cm}^{2}$ and a quasibound state lifetime of $50 \mathrm{fs}$. These characteristics are consistent with high-irequency analog and digital appiications, and suggest that further experimental development of RIT devices could lead to technologically interesting results, in addition to the novel physical principles that have already been demonstrated.
The authors would like to thank $D$. Ting for helpful discussions. This work was supported in part by the Ofice of Naval Research under Contract No. No0014-89-J-1141. Three of us (J.R.S., E.T.Y., and M.K.J.) would like to acknowledge financial support from the Wilhelm and Martina Lundgren Foundation, the AT\& T Foundation, and the Nat= ural Sciences and Engineering Research Council of Canada, respectively.

${ }^{i}$ R. Tsu and L. Esaki, Appl. Phys. Lett. 22, 562 (1973).

${ }^{2}$ E. R. Brown, T. C. L. G. Solner, C. D. Parker, W. D. Goodhue, and C. L. Chen, Appl. Phys. Lett. 35, 1777 (1989).

${ }^{3}$ S. Sen, F. Capasso, A. Y. Cho, and D. Sivco, IEEE Trans. Electron Devices ED3.34, 2185 (1987).

${ }^{4}$ R. Beresford, L. F. Luo, and W. I. Wang, Appl. Phys. Lett. 54, 1899 (1989).

${ }^{5}$ J. R. Söderström, D. H. Chow, and T. C. McGill, IEEE Electron Device Lett. EDL $11,27(1990)$.

${ }^{\circ} \mathrm{E}$. T. Yu and T. C. McGill, App1. Phys. Lett. 53, 60 (1988).

'M. Sweeny and J. Xu, Appl. Phys. Lett. 54, 546 (1989).

${ }^{8} J$. R. Söderström, D. H. Chow, and T. C. McGinl, Appl. Phys. Leti. 55, 1094 (1989).

${ }^{9}$ G. A. Sai-Halasz, R. Tsu, and L. Esaki, Appl. Phys. Lett. 30, 651 (1977).

${ }^{10} \mathrm{G}$. Bastard, Phys. Rew. B 24, 5693 (1981).

"G. Bastard, Phys. Rev. B 25, 7584 (1982).

${ }^{12}$ E. O. Kane, in Tuneling Phenomena in Solids, edited by E. Burstein and S. Lundqvist (Plenum, New York, 1969), p. 1.

"G. J. Gualtieri, G. B. Schwartz, R. G. Nuzzo, and W. A. Sunder, Appl. Phys. Lett. 49, 1037 (1986).

${ }^{14}$ G. A. Sai-Halasz, L. L. Chang, 3.-A. Welter, C.-A. Chang, and L. Esaki, Solid State Commun. 27, 935 (1978).

${ }^{15}$ G. 5. Gualtieri, G. P. Schwartz, R. G. Nuzzo, R. J. Malik, and J. F. Walker, J, Appl. Phys. 61, 5337 (1987).

16K. Taira, 1. Hase, and H. Kawa, Electro. Lett. 25, 1708 (1989).

\title{
Characterization of thin CdS films grown by the gradient recrystallization and growth technique
}

\author{
J. L. Rivera Hernâridez, J. M. Gracia-Jimenez, R. Silva González, \\ and G. Martinez Montes \\ Departamento de Fisica, ICUAP, Apdo, Postal J-48, Puebla, Pue. México
}

(Received 4 May 1989; accepted for publication 27 March 1990)

\begin{abstract}
For the first time the growth of polycrystalline CdS films by the gradient recrystallization and growth technique on silicon, quartz, and glass substrates is reported. X-ray diffraction, photoluminescence, scanning electron microscopy, and microprobe studies were used for their characterization. This preliminary study shows that this technique is suitable for growing CdS films with larger grain sizes than those obtained by conventional evaporation methods. The photoluminescence studies show that the emission spectrum of the films deposited on silicon is comparable to single-crystal CdS.
\end{abstract}

Cadmium sulfide is an important semiconductor with a direct energy band in the visible region of the electromagnetic spectrum; this property makes the material interesting for optical applications. Semiconducting CdS has been used as photoconductive, ${ }^{3}$ piezoelectric, ${ }^{2}$ and laser ${ }^{3}$ materiai. These CdS films have been grown on several amorphous and crystalline substrates by conventional evaporation, $\mathrm{CO}_{2}$ laser beam evaporation, ${ }^{4}$ sputtering, ${ }^{5}$ and spray pyrolysis, ${ }^{6}$ with 